\title{
Role of the renal circadian timing system in maintaining water and electrolytes
}

\section{homeostasis}

\author{
Dmitri Firsov $^{1}$, Natsuko Tokonami ${ }^{1}$, Olivier Bonny ${ }^{1,2}$
}

1 - Department of Pharmacology and Toxicology, University of Lausanne, 1005 Lausanne, Switzerland

${ }^{2}$ - Service of Nephrology, Department of Medicine, CHUV, 1011 Lausanne, Switzerland

to whom correspondence should be addressed:

\section{Dmitri Firsov}

Department of Pharmacology and Toxicology, University of Lausanne, 27 rue du Bugnon, 1005 Lausanne, Switzerland

Phone: ++ 41-216925406 Fax:++41-216925355 e-mail: dmitri.firsov@unil.ch

and to:

Olivier Bonny

Department of Pharmacology and Toxicology, University of Lausanne, 27 rue du Bugnon, 1005 Lausanne, Switzerland

Phone: ++ 41-216925417 Fax: ++41-216925355 e-mail: olivier.bonny@unil.ch 
Many basic physiological functions exhibit circadian rhythmicity. These functional rhythms are driven, in part, by the circadian clock, a ubiquitous molecular mechanism allowing cells and tissues to anticipate regular environmental events and to prepare for them. This mechanism has been shown to play a particularly important role in maintaining stability (homeostasis) of internal conditions. Because the homeostatic equilibrium is continuously challenged by environmental changes, the role of the circadian clock is thought to consist in the anticipative adjustment of homeostatic pathways in relation with the 24-hour environmental cycle. The kidney is the principal organ responsible for the regulation of the composition and volume of extracellular fluids (ECF). Several major parameters of kidney function, including renal plasma flow (RPF), glomerular filtration rate (GFR) and tubular reabsorption and secretion have been shown to exhibit strong circadian oscillations. Recent evidence suggest that the circadian clock can be involved in generation of these rhythms through external circadian time cues (e.g. humoral factors, activity and body temperature rhythms) or, trough the intrinsic renal circadian clock. Here, we discuss the role of renal circadian mechanisms in maintaining homeostasis of water and three major ions, namely, $\mathrm{Na}^{+}, \mathrm{K}^{+}$ and $\mathrm{Cl}^{-}$. 
The primary function of the kidney is to maintain the stability of the volume and the chemical composition of extracellular fluids (ECF). This is achieved through a combination of three basic renal mechanisms: glomerular filtration, tubular reabsorption and tubular secretion. Some of the filtered substances are totally reabsorbed along the nephron (amino acids, peptides, glucose, etc.), whereas others, after being adjusted by reabsorption and/or secretion, are excreted in the urine. Numerous physiological studies have shown that urinary excretion of water and major electrolytes $\left(\mathrm{Na}^{+}, \mathrm{K}^{+}, \mathrm{Cl}^{-}, \mathrm{H}^{+}, \mathrm{Ca}^{2+}\right.$ and phosphate) exhibit significant circadian rhythms (Aizman et al., 1994; Minors and Waterhouse, 1982; Roelfsema et al., 1980). Moreover, it has been demonstrated that many of these rhythms display self-sustained activity, thereby indicating an involvement of the circadian timing system (reviewed in (Firsov and Bonny, 2010) and in (Bonny and Firsov, 2009)). Dysregulation in renal excretory rhythms due to malfunction of the circadian clock or its misalignment with the imposed rest-activity or feeding cycles has been proposed as a possible cause of hypertension, renal stone formation and other serious long-term health problems (Sachdeva and Weder, 2006; Touitou et al., 1983). Here, we review the current knowledge regarding the role of the circadian timing system in maintaining homeostasis of water and major electrolytes, with a special focus on the homeostasis of ions, which are playing a major role in blood pressure control $\left(\mathrm{Na}^{+}, \mathrm{K}^{+}\right.$and $\mathrm{Cl}^{-}$ ). As most of the adjustments in urine volume and composition take place in the distal parts of the renal tubule, we focus on the role of the circadian timing system in the distal nephron, comprised of the distal convoluted tubule (DCT) and the connecting tubule (CNT) and, in the collecting duct. This discussion is largely based on the circadian expression profiles (transcriptomes) that became recently available for the DCT/CNT and the cortical collecting duct (CCD) (Zuber et al., 2009).

\section{Sodium}


Sodium is the major cation in ECF and accounts for almost half of the osmolality of the ECF. Thus, precise control of sodium excretion by the kidney is critically important for maintaining plasma osmolality, blood volume and blood pressure. As mentioned above, sodium excretion in the urine follows a well-defined circadian pattern with a peak in the first half of the activity phase and a trough during the biological night (Mills and Stanbury, 1952). The existence of these rhythms can be explained by a variety of partially opposed functional requirements, e.g.: (i) excess sodium ingested during the activity phase must be rapidly excreted, due to the lack of compartments capable of temporal accumulation of this ion; (ii) the reabsorption capacity of the kidney for sodium ions must be increased during the activity phase to compensate for the increase in the glomerular filtration rate (GFR), which has been shown to exhibit circadian rhythms in phase with the activity and sodium excretion rhythms; (iii) the physiological requirement for increased blood pressure during the activity phase (see below). Circadian rhythm of the blood pressure parallels oscillations of sodium excretion, showing maximal values during biological day and a $10 \%$ to $20 \%$ dip during the sleep phase (reviewed in (Burnier et al., 2007)). In humans, the nocturnal dipping in blood pressure appears to have an important physiological meaning. Indeed, a blunted nocturnal decline in blood pressure is associated with a significantly amplified risk of end-organs damages, including cardiovascular and cerebrovascular complications. Burnier et al., and Bankir et al., have proposed that nocturnal blood pressure is determined by the capacity of the kidney to excrete sodium during the daytime, and that the impairment in renal sodium excretory rhythms might be the principal cause of increased blood pressure in non-dipping patients (Bankir et al., 2008; Burnier et al., 2007). However, the molecular origin of these rhythms as well as the possible mechanisms of their dysfunction remain largely unknown. Recent data suggest that the circadian timing system could be one of the principal driving forces for these rhythms. Two mechanisms have been proposed: (i) entrainment of sodium reabsorption rhythms by external circadian time 
cues, such as the mineralocorticoid hormone aldosterone and, (ii) regulation of sodium reabsorption by the intrinsic renal circadian clock. Aldosterone is the main factor controlling sodium reabsorption in the distal nephron and the collecting duct. This hormone is released by the zona glomerulosa of the adrenal cortex and its principal role consists in the adjustment of the unitary conductance and the cell surface expression of the epithelial sodium channel $(\mathrm{ENaC})$, located in the distal part of the DCT, the CNT and the collecting duct (Rossier et al., 2002). Doi et al. have recently shown that the circadian clock controls plasma aldosterone levels by modulating expression of Hsd3b6, an enzyme involved in aldosterone synthesis in adrenal glands ((Doi et al., 2009), the role of molecular clock in aldosterone synthesis is discussed in more details in this issue). Wang et al. have shown that mice devoid of the clockcontrolled Par bZip circadian transcriptional factors Dbp, Hlf and Tef exhibit low aldosterone levels and low blood pressure values, thereby providing additional support for the role of the circadian clock in the control of aldosterone synthesis (Wang et al., 2010). However, several lines of evidence indicate that circadian regulation of plasma aldosterone levels is not the only circadian mechanism involved in the control of sodium reabsorption by the kidney. For instance, the cry $1 /$ cry 2 double knockout mice display a 10-fold increase in plasma aldosterone levels, but no difference in blood pressure upon normal salt diet (Doi et al., 2009). These observations indicate that deletion of cry $1 /$ cry 2 also results in a decreased capacity of the distal nephron and/or the collecting duct to reabsorb sodium. Interestingly in this regard, expression levels of $\alpha \mathrm{ENaC}$ subunit is significantly reduced in kidneys of perl knockout mice (Gumz et al., 2009). Whether this decrease in $\alpha \mathrm{ENaC}$ expression takes place in kidneys of $\mathrm{cry} 1 /$ cry 2 knockouts has not yet been tested. We have recently performed temporal RNA expression profiling of microdissected mouse DCT/CNT and CCD (Zuber et al., 2009). Analysis of the transcriptomes revealed that the principal elements of the circadian clock Bmal1, Clock, Npas2, Cry1, Cry2, Per1, Per2, Nr1d1, Dbp, Hlf and Tef exhibit high-amplitude circadian 
oscillations, thereby indicating that both DCT/CNT and CCD possess functional intrinsic clocks. Interestingly, none of the $\alpha, \beta$ or $\gamma \mathrm{ENaC}$ subunits showed evidence of circadian rhythmicity (assessed by a lack of fit to a cosine function). However, the same analysis revealed circadian oscillations in expression of a great number of genes engaged in a variety of regulatory pathways controlling the $\mathrm{ENaC}$ - mediated sodium transport (circadian profiles of expression of some of these transcripts are shown in Figure 1). For instance, significant circadian oscillations were observed in the alpha subunit of the thyroid hormone receptor (Thro). Thyroid hormones have been shown to modulate the activity of the Na,K-ATPase, which provides the driving force for the transepithelial sodium reabsorption (Morel and Doucet, 1986). The cell surface expression of the Na,K-ATPase is also dependent upon associations with arrestins 1 and 2 (Arrb2 and Arrb3, respectively), which display significant circadian oscillations both in the DCT/CNT and CCD (Kimura et al., 2007). Several circadian transcripts encode proteins involved in prostaglandins synthesis (PTGES, PTGES3, PLCG1, PLCG2, PLCB2), metabolism (HPGD) and signaling (PTGER4 and PTGFR). Prostaglandins PGE2 and PGF2 are synthesized in the DCT/CNT and CCD and act in a paracrine/autocrine manner on $\mathrm{ENaC}$ by regulating the open probability $\left(\mathrm{NP}_{\mathrm{o}}\right)$ of this channel (Wang et al., 2009). Previously, Ignatowska-Switalska showed the existence of circadian rhythms in the urinary excretion of PGE2 and PGF2 (Ignatowska-Switalska, 1983). Collectively, these results demonstrate that the effect of prostaglandins on ENaC could be variable depending on the circadian time and provide an example of a mechanism in which the circadian changes in $\mathrm{ENaC}$ activity does not necessarily require circadian changes in the expression of this channel at the RNA or the protein. The full activation of $\mathrm{ENaC}$ requires the cleavage of its subunits by serine proteases (Rossier and Stutts, 2009). Analysis of transcriptomes revealed that Prss8 (prostasin), a glycosylphosphatidylinositol-anchored serine protease with a well-defined role in the proteolytic cleavage of ENaC, displays a circadian rhythm of expression with a peak at the 
beginning of the activity phase. Interestingly, this rhythm fully parallels the circadian changes in $\mathrm{ENaC}$ activity (Wang et al., 2000). The residence time of $\mathrm{ENaC}$ at the cell surface is regulated by the ubiquitination and proteasomal degradation. It has been proposed that USP2, an ubiquitin-specific protease, can regulate this process by deubiquitinating the $\mathrm{ENaC}$ subunits (Fakitsas et al., 2007). In both, DCT/CNT and CCD, the USP2 transcript displays significant circadian oscillations. However, USP2 is ubiquitously expressed and exhibits circadian rhythms in all mouse tissues tested. Thus, the relevance of USP2 oscillations in the regulation of the ENaC activity remains unclear. Finally, a high-amplitude circadian rhythm was observed with Pkhd1, the gene mutated in human autosomal recessive polycystic kidney disease (ARPKD). Cortical collecting ducts isolated from ARPKD mice exhibit a significantly reduced transepithelial sodium transport and it was proposed that this reduction may contribute to the pathophysiology of ARPKD via accumulation of luminal fluids (Veizis et al., 2004). To our knowledge, this is the first demonstration of the involvement of a mutated circadian controlled gene in the pathogenesis of the polycystic renal disease.

Altogether, circadian oscillations in multiple regulators of $\mathrm{ENaC}$ activity indicate that circadian clocks may have a major influence on the transepithelial sodium reabsorption in the distal nephron and the collecting duct and, thus on blood pressure. Yet, an intriguing question about the relative role of the external circadian time-cues versus the intrinsic renal clocks in generation of these oscillations remains open. Recent genome-wide profiling of Bmall binding sites performed in the liver revealed that several of the circadian transcripts identified in DCT/CNT and/or CCD transcriptomes (Thro, Arrb2, Usp2 and Ptgfr) are the direct targets of the molecular clock (Hatanaka et al., 2010). In the kidney, this type of analysis has not yet been performed.

\section{Chloride}


It is well established that sodium chloride has a significantly greater effect on the blood pressure than other sodium salts. However, the mechanism(s) of interaction between the sodium and chloride reabsorption in the distal nephron and the collecting duct remained unclear until recently. The independent role of chloride in maintaining vascular volume and blood pressure was best demonstrated in the collecting duct, where sodium and chloride are reabsorbed through distinct transporter systems (reviewed in (Eladari et al., 2009)). Chloride enters into the B-type of intercalated cells via the apical electroneutral chloride/bicarbonate exchanger pendrin (Slc26a4) and leaves the cell at the basolateral side through the Clc-Kb chloride channel. However, the role of other, yet uncharacterized transporters cannot be excluded. It has been proposed that chloride is also reabsorbed via tight junctional complex composed of transmembrane proteins claudins, occludins and JAMs. Analysis of the DCT/CNT and CCD transcriptomes revealed two circadian transcripts (Slc26a2 and claudin-8) that encode proteins involved in chloride movement across the epithelia. The Slc26a2 is a multifunctional apical transporter, which transports chloride in exchange with sulfate, oxalate or other anions (Heneghan et al., 2010). The role of this transporter in renal chloride reabsorption has not been studied; however, a high expression level of Slc26a2 transcripts in the collecting duct indicates that this transporter may participate in the apical chloride movement. Claudin-8 is involved in the formation of a paracellular chloride channel located in the tight junctions of collecting duct cells (Hou et al., 2010). Thus, circadian oscillations in claudin-8 expression may provide a mechanism for circadian rhythmicity in the paracellular chloride permeability (Figure 1). A possible physiological meaning for the circadian rhythmicity of chloride reabsorption lies in the requirements for maintenance of the overall electroneutrality of $\mathrm{NaCl}$ transport across the cell under conditions when sodium reabsorption exhibits a clear circadian rhythm. 


\section{Potassium}

Potassium is the main cation of the intracellular compartment that contributes to the critical control of cell volume, intracellular $\mathrm{pH}$ and to a variety of enzymatic reactions. Low extracellular potassium concentration is critical for the establishment of the resting cell membrane potential and of neuromuscular and cardiac activities. Even if nowadays potassium ingestion is by far lower than during Paleolithic time (Eaton and Konner, 1985; Halperin et al., 2006), significant potassium intake may still represent a serious challenge to potassium homeostasis and need robust excretion paths. Indeed, several mechanisms are serving the body in eliminating supplemental potassium, starting from the still putative circulating kaliuretic factor to intestinal and renal excretion mechanisms. The kidney has the formidable capacity of excreting large amount of potassium in the distal parts (DCT, CNT, CD) of the tubules in a state of potassium repletion. Potassium excretion is dependent on filtration, on tubular flux, and on luminal secretion mechanisms mediated by ROMK1 (KCNJ1) and BK (KCNMA1 and $K C N M B 1)$ channels and regulated by aldosterone, vasopressin, insulin and $\mathrm{pH}$, among others (Hebert et al., 2005).

Circadian rhythms of urinary potassium excretion were reported many years ago (Moore Ede et al., 1975), with a peak in the middle of the day in humans. This rhythm is independent of activity, posture and dietary intake and persists for more than several days in individuals isolated from main external cues. By using the transtubular potassium gradient as surrogate marker for evaluating potassium concentrations in the CCD, potassium concentration and not the tubular flow was found to be driving the cycling of potassium secretion (Steele et al., 1994). However, not much is known about the molecular mechanisms dragging these rhythms. Analysis of the rhythmicity of transcripts of the mouse DCT/CNT and CCD (Zuber et al., 2009) has unveiled several oscillating potassium channels, such as KCNJ1 (ROMK1 or Kir1.1), KCNK1 (Twik-1), KCNJ10 (Kir1.2). Amplitude of the oscillations varied between 30- 
$40 \%$ and the peak was at ZT20 for most of them, indicating a maximal expression level in the second part of the active period of the day in mice, in phase with the excretion of the bulk of potassium in the mouse (Firsov, unpublished). Finding that ROMK1 is cycling by about 30\% at the mRNA level might explain part of the circadian variation observed in urine potassium excretion.

\section{Water}

Water movement across biological membranes is a passive process driven by the difference in osmotic pressure. However, water permeability of the distal nephron and the collecting duct requires an additional stimulus. This stimulus is the antidiuretic hormone vasopressin, released by the pituitary gland in the brain in response to the increased plasma osmolality. In the kidney, vasopressin binds to the type 2 vasopressin receptor (V2R) located on the basolateral membrane of the distal nephron and of the collecting duct cells (Figure 1). The signaling cascade activated upon this binding stimulates the insertion in the apical membrane of the aquaporin-2 water channel, which represents the rate-limiting step for water entry into the cell. The basolateral water transport is mediated by the aquaporins -3 and -4 . Dysfunction in this mechanism leads to the nephrogenic diabetes insipidus (NDI), a disease characterized by the inability of the kidney to concentrate urine. In inherited NDI cases, the disease is caused either by mutations in the V2R gene $(\sim 90 \%)$ or in the aquaporin-2 gene $(\sim 10 \%)$. The rhythmicity in the renal water handling reflects the requirement to maintain plasma osmolality within narrow limits despite a multitude of interfering factors, e.g. (i) excess water ingested during the activity phase, (ii) circadian variations in the GFR, the renal sodium handling and in the extrarenal water losses. The importance of the circadian timing system in generation of these rhythms was evidenced in the Clock knockout mice, which exhibit an NDI-like phenotype characterized by the excretion of diluted urine and by the partial loss of circadian rhythmicity 
of urine excretion ((Zuber et al., 2009) and D. Firsov, unpublished observations). Analysis of DCT/CNT and CCD transcriptomes revealed that V2R, aquaporin-2 and aquaporin-4 expression follow synchronized circadian patterns with a maximum in the second half of the activity phase (Figure 1). The molecular origin of these rhythms remains uncharacterized. The putative circadian variations in plasma vasopressin levels are difficult to detect due to the low circulating levels of this hormone. Recently, the promoter region of the aquaporin-2 gene was shown to contain three E-boxes that can bind Bmal1/Clock or Bmal1/Npas2 heterodimers (Gumz et al., 2010). Thus, a direct regulation of aquaporin-2 expression by the intrinsic renal clock cannot be excluded.

In conclusion, recent data demonstrate that a great number of genes crucially involved in the renal control of water and electrolytes balance exhibit circadian expression patterns. Further in vivo studies are required to establish the link between the rhythms of transcript expression and the renal excretory rhythms. Most interesting in this regard appear the experiments with the nephron segment-specific deletion of the circadian clock system.

\section{ACKNOWLEDGMENTS}

DF is supported by a Swiss National Science Foundation grant \#31003A-132496. OB is supported by the Foundation Prof. Placide Nicod and a bridge grant of the Faculte de Biologie et Médecine of the University of Lausanne. 
Aizman, R.I., Rabinowitz, L. and Mayer-Harnisch, C., 1994. Circadian rhythms and time course of adaptive sodium and potassium excretion in rats after uninephrectomy. Am. J. Physiol. 266, R1454-1462.

Bankir, L., Bochud, M., Maillard, M., Bovet, P., Gabriel, A. and Burnier, M., 2008. Nighttime blood pressure and nocturnal dipping are associated with daytime urinary sodium excretion in African subjects. Hypertension. 51, 891-898.

Bonny, O. and Firsov, D., 2009. Circadian clock and the concept of homeostasis. Cell Cycle. 8, 4015-4016.

Burnier, M., Coltamai, L., Maillard, M. and Bochud, M., 2007. Renal sodium handling and nighttime blood pressure. Semin. Nephrol. 27, 565-571.

Doi, M., Takahashi, Y., Komatsu, R., Yamazaki, F., Yamada, H., Haraguchi, S., Emoto, N., Okuno, Y., Tsujimoto, G., Kanematsu, A., Ogawa, O., Todo, T., Tsutsui, K., van der Horst, G.T. and Okamura, H., 2009. Salt-sensitive hypertension in circadian clock-deficient Cry-null mice involves dysregulated adrenal Hsd3b6. Nat. Med. 16, 67-74.

Eaton, S.B. and Konner, M., 1985. Paleolithic nutrition. A consideration of its nature and current implications. N Engl J Med. 312, 283-289.

Eladari, D., Chambrey, R., Frische, S., Vallet, M. and Edwards, A., 2009. Pendrin as a regulator of ECF and blood pressure. Curr. Opin. Nephrol. Hypertens. 18, 356-362.

Fakitsas, P., Adam, G., Daidie, D., van Bemmelen, M.X., Fouladkou, F., Patrignani, A., Wagner, U., Warth, R., Camargo, S.M., Staub, O. and Verrey, F., 2007. Early aldosterone-induced gene product regulates the epithelial sodium channel by deubiquitylation. J. Am. Soc. Nephrol. 18, 1084-1092.

Firsov, D. and Bonny, 0., 2010. Circadian regulation of renal function. Kidney International. 78, 640-645.

Gumz, M.L., Cheng, K.Y., Lynch, I.J., Stow, L.R., Greenlee, M.M., Cain, B.D. and Wingo, C.S., 2010. Regulation of alphaENaC expression by the circadian clock protein Period 1 in mpkCCD(c14) cells. Biochim. Biophys. Acta. 1799, 622629.

Gumz, M.L., Stow, L.R., Lynch, I.J., Greenlee, M.M., Rudin, A., Cain, B.D., Weaver, D.R. and Wingo, C.S., 2009. The circadian clock protein Period 1 regulates expression of the renal epithelial sodium channel in mice. J. Clin. Invest. 119, 2423-2434.

Halperin, M.L., Cheema-Dhadli, S., Lin, S.H. and Kamel, K.S., 2006. Control of potassium excretion: a Paleolithic perspective. Curr. Opin. Nephrol. Hypertens. 15, 430-436.

Hatanaka, F., Matsubara, C., Myung, J., Yoritaka, T., Kamimura, N., Tsutsumi, S., Kanai, A., Suzuki, Y., Sassone-Corsi, P., Aburatani, H., Sugano, S. and Takumi, T., 2010. Genome-wide profiling of the core clock protein BMAL1 targets reveals a strict relationship with metabolism. Mol. Cell. Biol. 30, 5636-5648.

Hebert, S.C., Desir, G., Giebisch, G. and Wang, W., 2005. Molecular diversity and regulation of renal potassium channels. Physiol. Rev. 85, 319-371.

Heneghan, J.F., Akhavein, A., Salas, M.J., Shmukler, B.E., Karniski, L.P., Vandorpe, D.H. and Alper, S.L., 2010. Regulated transport of sulfate and oxalate by SLC26A2/DTDST. Am. J. Physiol. Cell. Physiol. 298, C1363-1375. 
Hou, J., Renigunta, A., Yang, J. and Waldegger, S., 2010. Claudin-4 forms paracellular chloride channel in the kidney and requires claudin-8 for tight junction localization. Proc. Natl. Acad. Sci. U S A. 107, 18010-18015.

Ignatowska-Switalska, H., 1983. Circadian rhythm of PGE2, PGF2 alpha and 6-ketoPGF1 alpha urinary excretion in healthy women. Prostaglandins Leukot. Med. 11, 233-240.

Kimura, T., Allen, P.B., Nairn, A.C. and Caplan, M.J., 2007. Arrestins and spinophilin competitively regulate $\mathrm{Na}+, \mathrm{K}+-\mathrm{ATPase}$ trafficking through association with a large cytoplasmic loop of the Na+,K+-ATPase. Mol. Biol. Cell. 18, 45084518.

Mills, J.N. and Stanbury, S.W., 1952. Persistent 24-hour renal excretory rhythm on a 12-hour cycle of activity. J. Physiol. 117, 22-37.

Minors, D.S. and Waterhouse, J.M., 1982. Circadian rhythms of urinary excretion: the relationship between the amount excreted and the circadian changes. J. Physiol. 327, 39-51.

Moore Ede, M.C., Brennan, M.F. and Ball, M.R., 1975. Circadian variation of intercompartmental potassium fluxes in man. J. Appl. Physiol. 38, 163-170.

Morel, F. and Doucet, A., 1986. Hormonal control of kidney functions at the cell level. Physiol. Rev. 66, 377-468.

Roelfsema, F., van der Heide, D. and Smeenk, D., 1980. Circadian rhythms of urinary electrolyte excretion in freely moving rats. Life Sci. 27, 2303-2309.

Rossier, B.C. and Stutts, M.J., 2009. Activation of the epithelial sodium channel (ENaC) by serine proteases. Annu. Rev. Physiol. 71, 361-379.

Rossier, B.C., Pradervand, S., Schild, L. and Hummler, E., 2002. Epithelial sodium channel and the control of sodium balance: interaction between genetic and environmental factors. Annu. Rev. Physiol. 64, 877-897.

Sachdeva, A. and Weder, A.B., 2006. Nocturnal sodium excretion, blood pressure dipping, and sodium sensitivity. Hypertension. 48, 527-533.

Steele, A., deVeber, H., Quaggin, S.E., Scheich, A., Ethier, J. and Halperin, M.L., 1994. What is responsible for the diurnal variation in potassium excretion? Am. J. Physiol. 267, R554-560.

Touitou, Y., Touitou, C., Charransol, G., Reinberg, A., Thomas, J., Bogdan, A., Barthelemy, C. and Desgrez, P., 1983. Alterations in circadian rhythmicity in calcium oxalate renal stone formers. Int. J. Chronobiol. 8, 175-192.

Veizis, E.I., Carlin, C.R. and Cotton, C.U., 2004. Decreased amiloride-sensitive Na+ absorption in collecting duct principal cells isolated from BPK ARPKD mice. Am. J. Physiol. Renal Physiol. 286, F244-254.

Wang, Q., Maillard, M., Schibler, U., Burnier, M. and Gachon, F., 2010. Cardiac hypertrophy, low blood pressure, and low aldosterone levels in mice devoid of the three circadian PAR bZip transcription factors DBP, HLF, and TEF. Am. J. Physiol. Regul. Integr. Comp. Physiol. 299, R1013-1019.

Wang, Q., Horisberger, J.D., Maillard, M., Brunner, H.R., Rossier, B.C. and Burnier, M., 2000. Salt- and angiotensin II-dependent variations in amiloride-sensitive rectal potential difference in mice. Clin. Exp. Pharmacol. Physiol. 27, 60-66.

Wang, S., Meng, F., Xu, J. and Gu, Y., 2009. Effects of lipids on ENaC activity in cultured mouse cortical collecting duct cells. J. Membr. Biol. 227, 77-85.

Zuber, A.M., Centeno, G., Pradervand, S., Nikolaeva, S., Maquelin, L., Cardinaux, L., Bonny, O. and Firsov, D., 2009. Molecular clock is involved in predictive 
circadian adjustment of renal function. Proc. Natl. Acad. Sci. U S A. 106, 16523-16528. 
urine space

blood
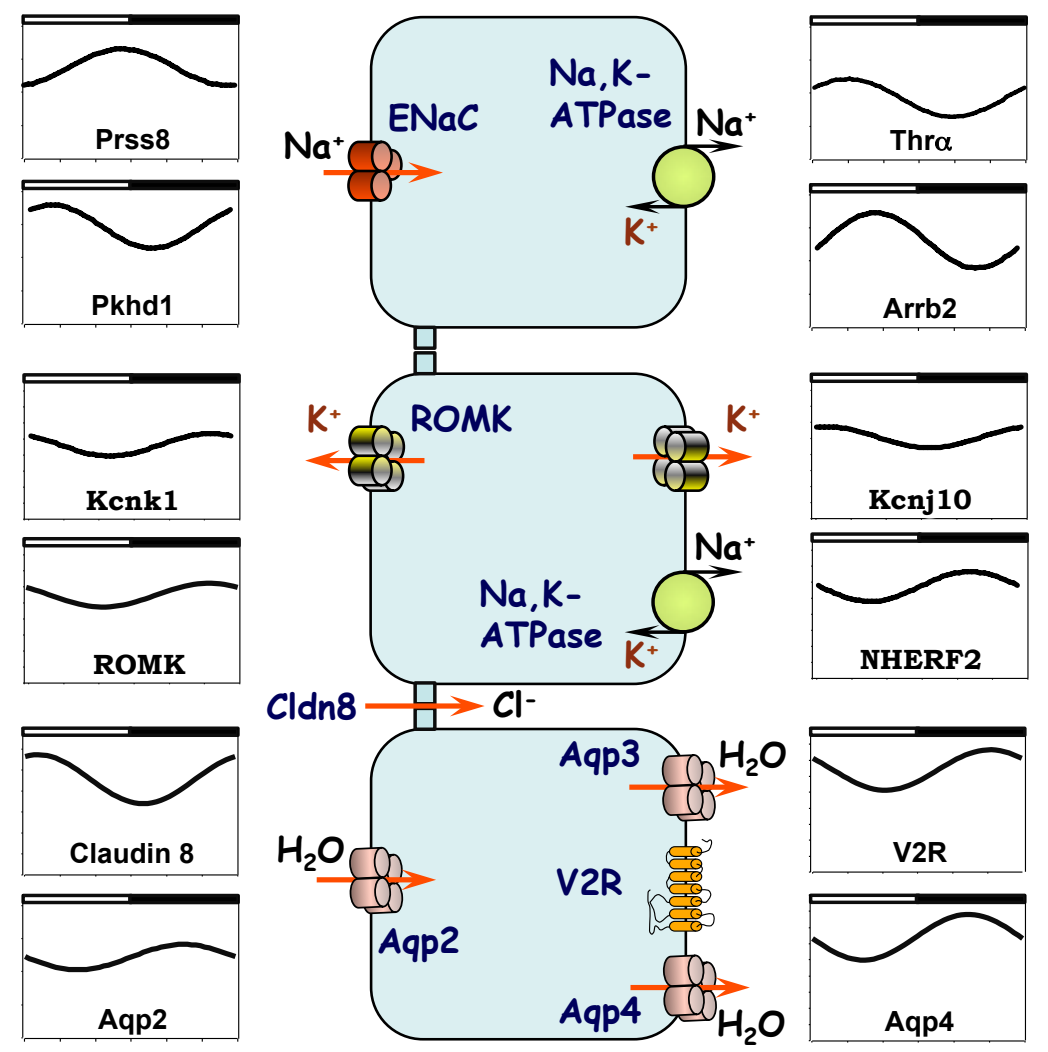

Figure 1. Circadian expression patterns of selected genes involved in the renal control of water and electrolyte balance (Zuber, Centeno, Pradervand et al., 2009). The Y-axis shows the RNA expression levels in arbitrary units. The X-axis represents a period of one 24-hour circadian cycle; the white bar on the top of the graphs represents the period of light (0-12 hours); the black bar on the top of the graphs represents the period of dark (12-24 hours). Temporal expression profiles of the represented transcripts fit to a cosine function with the false discover rate $(\mathrm{FDR})<0.1$. 